\title{
The GALEX mission and detectors
}

Oswald H. W. Siegmund, Barry Y. Welsh, Christopher Martin, Thomas Barlow, Luciana Bianchi, et al.

Oswald H. W. Siegmund, Barry Y. Welsh, Christopher Martin, Thomas Barlow, Luciana Bianchi, Yong-Ik Byun, Karl Forster, Peter G. Friedman, Patrick N. Jelinsky, Barry F. Madore, Roger Malina, Bruno Milliard, Patrick F. Morrissey, Susan G. Neff, Michael R. Rich, David Schiminovich, Todd Small, Alexander Szalay, Ted K. Wyder, "The GALEX mission and detectors," Proc. SPIE 5488, UV and Gamma-Ray Space Telescope Systems, (11 October 2004); doi: 10.1117/12.561488

Event: SPIE Astronomical Telescopes + Instrumentation, 2004, Glasgow, United Kingdom 


\title{
The GALEX mission and detectors
}

\author{
Oswald Siegmund*g ${ }^{\mathrm{g}}$, Barry Welsh ${ }^{\mathrm{g}}$, Christopher Martin ${ }^{\mathrm{a}}$, Tom Barlow ${ }^{\mathrm{a}}$, Luciana Bianchi ${ }^{\mathrm{d}}$, Yong-Ik \\ Byun $^{\mathrm{h}}$, Karl Forster ${ }^{\mathrm{a}}$, Peter G. Friedman ${ }^{\mathrm{a}}$, Patrick Jelinsky ${ }^{\mathrm{g}}$, Barry Madore ${ }^{\mathrm{f}}$, Roger Malina ${ }^{\mathrm{g}, \mathrm{c}}$, Bruno \\ Milliard $^{\mathrm{c}}$, Patrick Morrissey ${ }^{\mathrm{a}}$, Susan Neff ${ }^{\mathrm{i}}$, Mike Rich ${ }^{\mathrm{e}}$, David Schiminovich ${ }^{\mathrm{a}}$, Todd Small ${ }^{\mathrm{a}}$, Alex \\ Szalay ${ }^{\mathrm{d}}$, Ted Wyder ${ }^{\mathrm{a}}$.
}

${ }^{a}$ California Institute of Technology, ${ }^{b} \mathrm{JPL},{ }^{\mathrm{c}}$ Laboratoire Astrophysique Marseille, ${ }_{\mathrm{d}}^{\mathrm{d}}$ Johns Hopkins University, ${ }^{\mathrm{e}}$ UCLA, ${ }^{\mathrm{f}}$ Carnegie Institute of Washington, ${ }^{\mathrm{g}} \mathrm{U}$.C.Berkeley, ${ }^{\mathrm{h}}$ Yonsei University, ${ }^{\mathrm{i}}$ NASA-Goddard Space Flight Center,

\begin{abstract}
We describe the Galaxy Evolution Explorer (GALEX) satellite that was launched in April 2003 specifically to accomplish far ultraviolet (FUV) and near ultraviolet (NUV) imaging and spectroscopic sky-surveys. GALEX is currently providing new and significant information on how galaxies form and evolve over a period that encompasses $80 \%$ of the history of the Universe. This is being accomplished by the precise measurement of the UV brightness of galaxies which is a direct measurement of their rate of star formation. We briefly describe the design of the GALEX mission followed by an overview of the instrumentation that comprises the science payload. We then focus on a description of the development of the UV sealed tube micro-channel plate detectors and provide data that describe their on-orbit performance. Finally, we provide a short overview of some of the science highlights obtained with GALEX.
\end{abstract}

Keywords: GALEX, far ultraviolet, near ultraviolet, microchannel plates, imaging, spectroscopy

\section{INTRODUCTION}

The Galaxy Evolution Explorer (GALEX) satellite ${ }^{1,2}$ is a NASA Small Explorer mission currently on-orbit and carrying out the first all-sky deep imaging and spectroscopic surveys at near and far ultraviolet wavelengths (1350 $2800 \AA$ ). The GALEX instrument employs a $50 \mathrm{~cm}$ aperture, modified Ritchey-Chretien telescope, a dichroic beamsplitter and two $65 \mathrm{~mm}$ diameter sealed tube microchannel plate detectors that simultaneously cover the 1.2 degree field of view in two ultraviolet bands. The scientific instrument is coupled to a three-axis stabilized spacecraft bus built by Orbital Sciences Corporation (OSC), and the satellite was launched via an OSC Pegasus-XL vehicle into a 29 degree inclination, $690 \mathrm{~km}$ circular orbit on April 28, 2003. After a two month in-orbit checkout period GALEX began nominal operations. The initial phase of the mission consists of performing 5 imaging surveys in each of two bands $(1350-1750 \AA$ and $1750-2800 \AA)$ that cover areas ranging from an all-sky survey (of limiting magnitude $\mathrm{m}_{\mathrm{v}} \sim 21$ ) to an ultra-deep survey that covers only 4 square degrees to a limiting magnitude of $\mathrm{m}_{\mathrm{v}} \sim 26$. In addition GALEX is also performing three low resolution $(\mathrm{R}=100-$ 300) spectroscopic grism surveys with various depths and sky coverage over the entire $1350-2800 \AA$ wavelength band. These ultraviolet survey data will address the primary science goals of the GALEX mission which are (a) to determine the star formation history and evolution of the local Universe and (b) to measure the UV properties of local galaxies. At present about $30 \%$ of these surveys have been completed and the GALEX instrument and spacecraft are operating consistently well ${ }^{3}$. Starting October 1, 2004 one third of mission time will be devoted to Guest Investigator observations, while the remaining observing time will be devoted to completing the various surveys.

\section{MISSION}

All GALEX science data are obtained only on the night side of the orbit. During the day side of the orbit the satellite solar panels face toward the Sun. As the satellite enters twilight, it slews to one of the pre-selected survey target areas. The imaging window or grism can be selected for imaging or spectroscopic targets. If the target is spectroscopic, the grism rotation may also be selected. The satellite attitude is dithered in a 1 arcminute spiral for deep targets, while the all-sky survey is obtained by scanning at a rate of 200 arcseconds/sec. Dithering and scanning is performed to average over detector non-uniformities and to prevent microchannel plate detector gain fatigue by UV bright stars.

*Email: ossy@ssl.berkeley.edu, Phone 510-642-0895 
During science data collection, individual photon events are collected by the far ultraviolet and near ultraviolet detectors and front-end electronics (FEE), formatted by the instrument Digital Processing Unit (DPU), and stored on the spacecraft solid-state recorder (SSR) along with housekeeping data.

At the end of orbital night, detector high voltages are ramped to idle levels to protect them from damage and the spacecraft returns to solar array pointed attitude. Up to four times per 24 hour day the SSR is dumped via the X-band transmitter to ground stations in Hawaii or Perth, Australia, which are operated by Universal Space Networks (USN). Realtime satellite health and safety monitoring is performed by the Mission Operations Center (MOC) at Orbital Sciences Corporation in Dulles, Virginia, during the ground pass. Science telemetry is shipped by ground network to the GALEX Science Operations Center (SOC) at the California Institute of Technology (Caltech) with a latency of 4 hours for housekeeping and 48 hours for photon data. Science data is processed at the SOC to produce images, object catalogs, and extracted spectra. Catalogs and spectra will eventually be delivered to the Space Telescope Science Institute to be archived in a public database developed by Johns Hopkins University for the Sloan Digital Sky Survey (SDSS).

\section{INSTRUMENT}

The design of GALEX provides $\sim 5$ arc-second FWHM resolution imaging with a high throughput, large circular field of view (65 mm or $1.24 \mathrm{deg}$ ), in four different optical paths (two simultaneous near and far UV channels, with selectable imaging or slitless spectroscopy modes), while keeping the instrument compact and comparatively simple. The instrument overall layout is depicted in Figure 1, and the satellite is shown in Figure 2.

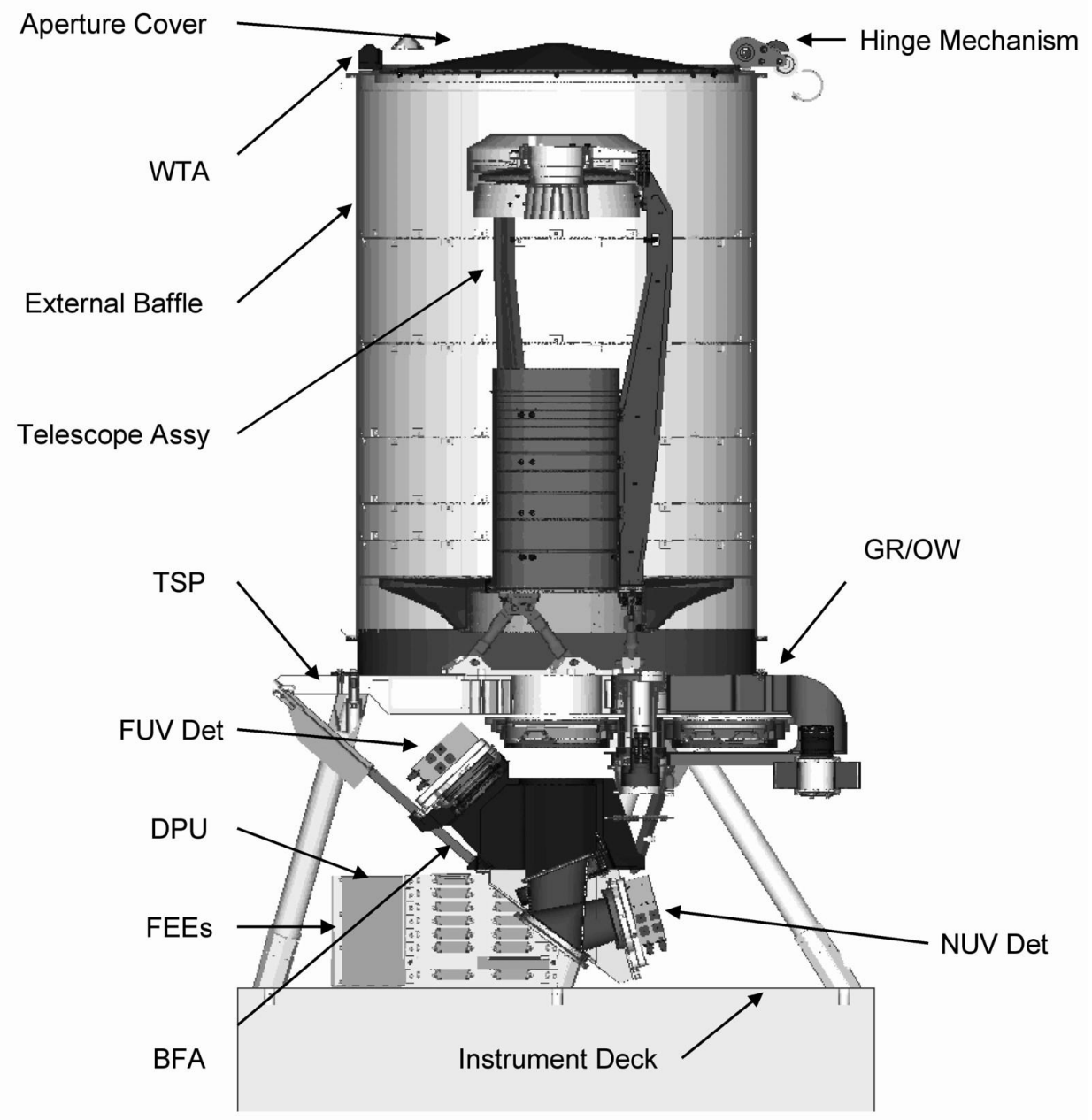

Figure 1. GALEX instrument layout showing all major components. 


\title{
3.1 Instrument Summary
}

\author{
NASA Small Explorer Mission ( 100M\$) \\ $50 \mathrm{~cm}$ aperture ultraviolet telescope \\ Large area $(65 \mathrm{~mm})$ ultraviolet photon-counting detectors \\ Imaging and slitless grism spectroscopy

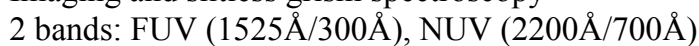 \\ Field of view: 1.24 degrees diameter \\ PSF diameter: 4-5" FWHM \\ Spectral resolution, R: 100-250 \\ Science observations began mid-June 2003
}

3.2 The UV Telescope: Ultraviolet photons are collected over a 1.24 degree field of view using a $50 \mathrm{~cm}$ diameter astigmatism corrected Ritchey-Chretien f/6 telescope built by Light Works Optical Corporation. The f/2 primary and secondary fused silica mirrors were overcoated with $\mathrm{Al}$ and $340 \AA$ of $\mathrm{MgF}_{2}$ by the NASA GSFC coating laboratory to enhance UV reflectivity.

3.3 The Window and Grism: The various observational modes (i.e. imaging, grism spectroscopy or blocked) are selected using a rotating optics wheel with a $\mathrm{CaF}_{2}$ imaging window, a 75 groove $/ \mathrm{mm} \mathrm{CaF}_{2}$ transmission grism and an opaque position. The grism was developed jointly by Jobin-Yvon and the Laboratoire d'Astrophysique de Marseilles and achieves unprecedented efficiencies of $82 \%$ in the NUV and $61 \%$ in the FUV. The entire grism assembly can be rotated to 872 angles to achieve different position angles for recording the dispersed spectra during each orbit of integration. This allows the confusion caused by the numerous overlapping spectra (and orders) for each stellar object (which are produced in the slitless spectroscopy mode) to be minimized for easier subsequent data analysis.

3.4 The Dichroic Beamsplitter: Both FUV and NUV bands are simultaneously obtained using a 110mm diameter fused silica dichroic window, which also performs the task of field aberration correction. This beamsplitter/asphere is ion-etched with aspheric surfaces on both sides. The splitting of the input beam is accomplished via a dielectric multilayer deposited on the input side of the window which reflects the FUV band $(1350-1750 \AA)$ and transmits the NUV band $(1750-$ $2750 \AA)$.

3.5 The UV Filters: Three of the GALEX back focal assembly optics are coated with multilayer filters designed to enhance the in-band throughput and out-of-band rejection of the GALEX instrument. The dichroic coating on the beamsplitter achieves a reflectance of $\sim 61 \%$ in the FUV band and a mean transmittance of $\sim 83 \%$ at NUV wavelengths. A transmissive blue-edge filter coated on $\mathrm{MgF}_{2}$ provides $10 \%$ rejection of the strong OI $1304 \AA$ airglow line for the FUV channel. A reflective broad-band red-blocking filter on the beam-folding mirror has an edge at $2800 \AA$ which yields an additional factor of $10-20$ rejection for the NUV zodiacal light background in addition to that provided by the $\mathrm{Cs}_{2} \mathrm{Te}$ photocathode cut-off of the NUV detector $(\sim 3000 \AA)$.

3.6 Mechanics and Mechanisms: The GALEX instrument's mechanical hardware consists of a Telescope Support Plate (TSP), a Back Focal Assembly (BFA) and a Barrel Baffle with bipod supports. The TSP is the central node of the science instrument to which all other components are attached and it plays an integral part of the athermalization design of the telescope assembly and the BFA. The BFA design is athermalized using aluminum for the bench and invar for the metering structure in order to maintain telescope alignment in the presence of either a uniform temperature change or uniform temperature gradient.

GALEX has two primary mechanism assemblies, the Cover Assembly and the Grism Rotator/Optical Wheel (GR/OW). The Cover Assembly was a one-time deployable device that prevented contamination of the optical cavity during ground integration, testing and launch. It deployed successfully once on-orbit using a spring loaded hook and pin device that was freed from a latch assembly using a wax thermal actuator. The GR/OW consists of a grism rotator on an optical wheel that swaps between imaging and grism observing modes. The wheel can move 360 degrees in 872 steps using a mini-dual drive assembly that consists of a fully redundant brushless DC motor. The grism rotator mechanism uses a vacuum compatible stepper motor with an angle encoder. 


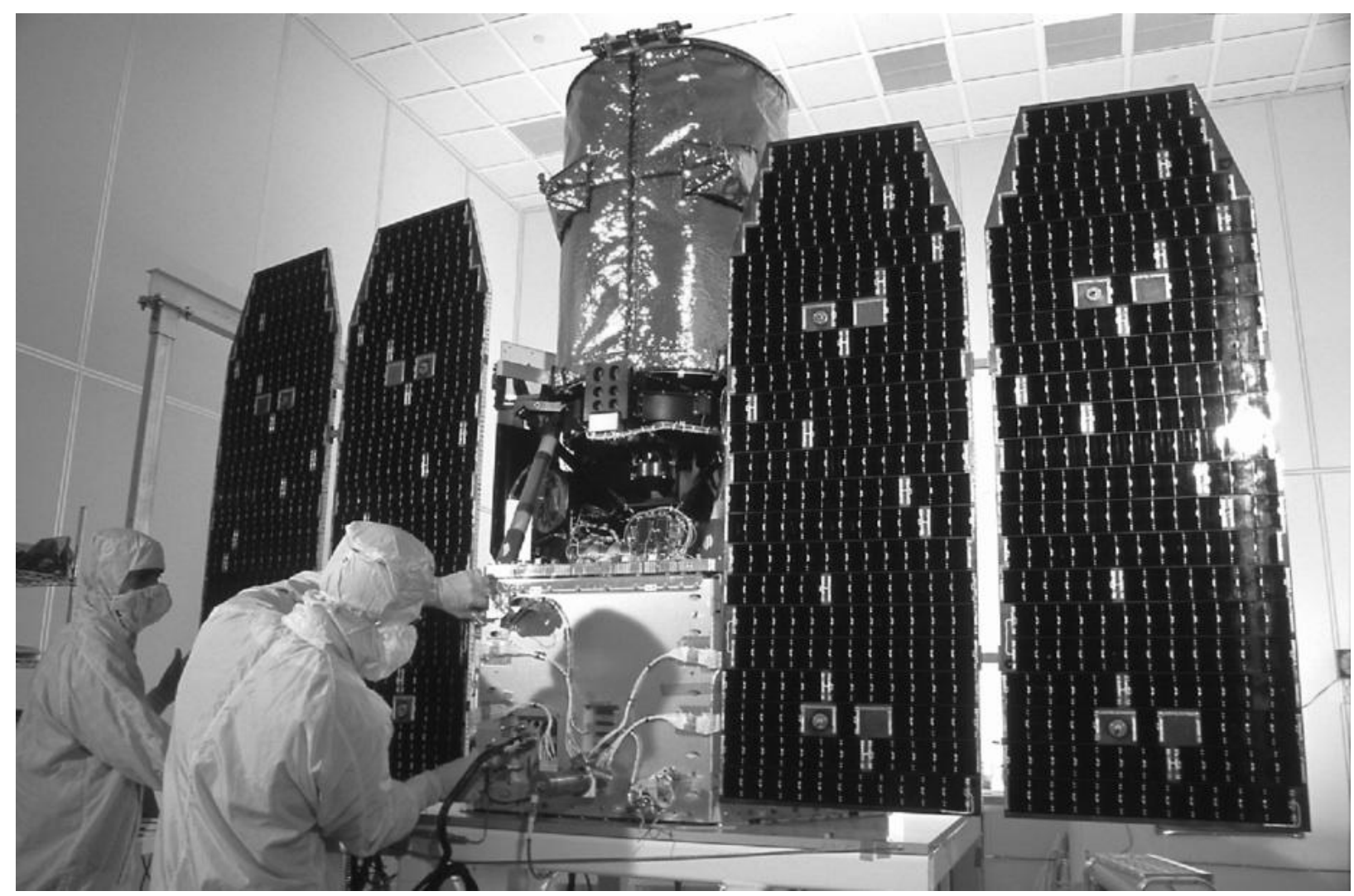

Figure 2. GALEX instrument and solar panels

\section{SCIENCE DETECTORS}

The GALEX detectors are large format (65mm diameter; 4096x4096 pixel) sealed tube microchannel plate (MCP) detectors with cross delay anode readouts ${ }^{4,5}$. The $65 \mathrm{~mm}$ diameter active area is the largest format sealed tube MCP detector currently on orbit. GALEX employs two sealed tube detectors, an NUV and an FUV, to achieve its desired wavelength coverage. The FUV detector has a spectral bandpass from $1150-1800 \AA$ and the NUV detector has a bandpass from 1650 $-3000 \AA$. Characteristics that have been measured include the background count rate and distribution, gain versus applied high voltage, spatial resolution and linearity, flat fields, and quantum efficiency. The detector tubes have been developed and built by the Experimental Astrophysics Group (EAG) at the University of California, Berkeley in collaboration with members of the Space Astrophysics Laboratory at Caltech. The two detectors are almost identical except for the window material and photocathode chosen for the spectral bandpass. The FUV tube has a $\mathrm{MgF}_{2}$ window and a CsI photocathode deposited onto the MCP. The NUV tube has a fused silica window deposited with a semitransparent $\mathrm{Cs}_{2} \mathrm{Te}$ photocathode proximity-focused to the MCP. Figure 3 is a photo of the NUV flight tube. The photons pass through the entrance window and interact with a photocathode, resulting in photoelectron emission. The MCP stack then multiplies the charge through an electron avalanche to a total gain of about $10^{7}$ electrons. This output charge cloud is accelerated ( $700 \mathrm{v}$ bias $)$ across a 6 $\mathrm{mm}$ gap to the cross delay line (XDL) anode (Figure 4). The charge is divided by the two delay line electrode sets so that $\mathrm{X}$ and $\mathrm{Y}$ event positions can be determined from the differences in signal arrival time at the two ends of the anode. The delay line detector scheme used is similar to those EAG has built and successfully flown on the $\mathrm{SOHO}^{6}$ and $\mathrm{IMAGE}^{7}$ missions.

For each detector, signals from the anode are processed by two identical separate electronic chains, one each for the $\mathrm{X}$ and $\mathrm{Y}$ positions. The position encoding electronics begins with fast timing amplifiers mounted on the rear of each detector. The amplifier signals are then sent to time to digital converters (TDC) for each axis. Constant fraction discriminators are used to produce timing signals for each end of the delay line axes, a start from one end, and a stop from the other end which is delayed by a specific length of cable. The time difference between the start and stop signals for each axis are converted to digital positions via a coarse/fine system. A counter is used to determine the number of clock cycles between the start and stop signals to give the 3 most significant bits of the coordinate. Then start and stop interpolators measure the fine position values for each signal relative to the clock. The counter, fine position values, and charge amplitude are then 
passed to the instrument. The $\mathrm{x} \& \mathrm{Y}$ positions are determined on the ground from the counter and fine position value data. The TDC's, low voltage power supplies, high voltage power supplies and interface electronics are co-located (Figure 1) as a Front End Electronics (FEE) stack close to the detectors.

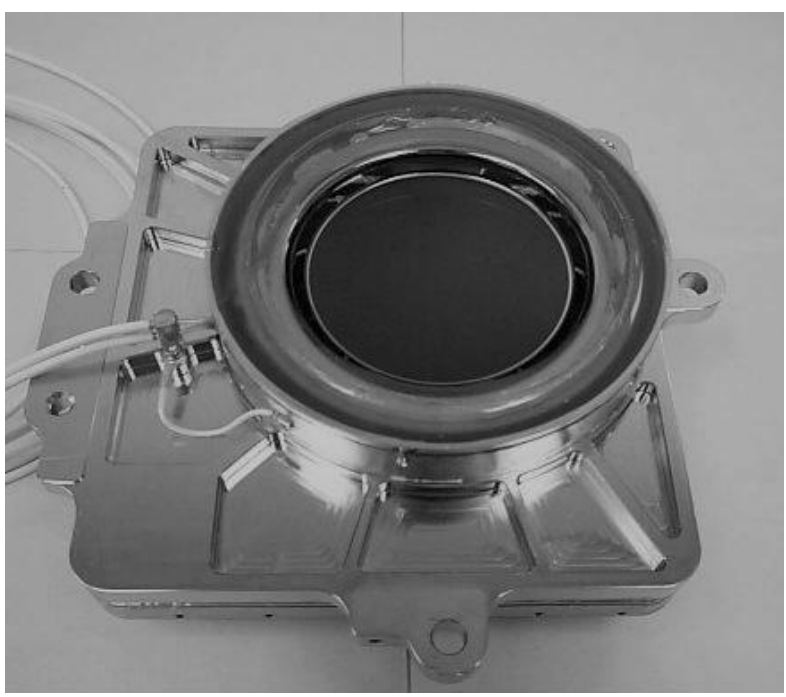

Figure 3. GALEX NUV sealed tube detector.

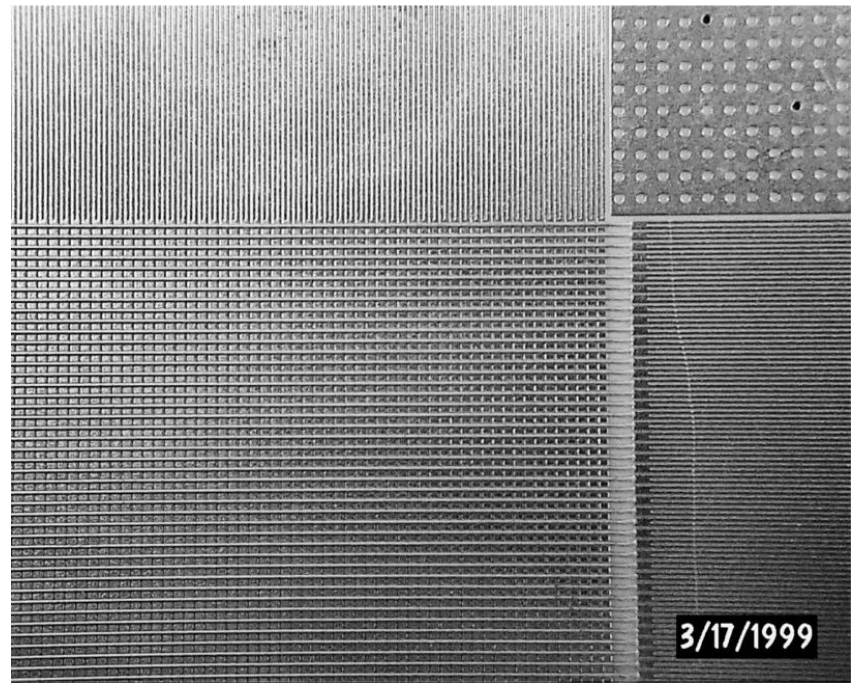

Figure 4. Portion of the GALEX cross delay line anode.

\subsection{Detector Performance}

The microchannel plates used were obtained from Photonis in sets of three, with $10 \mu \mathrm{m}$ and $12 \mu \mathrm{m}$ pore diameters and 80:1 channel length to diameter ratio. The tubes are constructed with three MCP's stacked in a "Z" configuration, and are typically operated at a gain between $10^{7}$ and $2 \times 10^{7}$ to optimize the imaging performance. The pulse height distributions are about $100 \%$ FWHM for the tubes, mostly as a result of gain variations across the large field of view.

After the tubes were sealed the final background (dark) rate was measured. Figure 5 shows the background image for the NUV tube just before delivery. The NUV tube background was probably dominated by room light leakage. Even though the $\mathrm{Cs}_{2} \mathrm{Te}$ cathode is solar blind, it still is responsive to room light. Prior to launch the rate of hotspots was about 100 counts/second and the diffuse rate was about 0.9 counts $/ \mathrm{sec} / \mathrm{cm}^{2}$. The FUV tube background is dominated by hotspots at about 20 counts/second, the diffuse rate was about 0.3 counts $/ \mathrm{sec} / \mathrm{cm}^{2}$, and there were about 7 counts $/$ second of field emission. In orbit the hotspot activity has remained about the same but the diffuse rate has increased in both detectors by about 0.5 counts $/ \mathrm{sec} / \mathrm{cm}^{2}$ due to the radiation environment, which is normal for experiments in this orbit. There is no significant contribution due to phosphorescence of the windows. These were carefully specified and measured beforehand to eliminate this potential problem. By far the greatest source of background is the sky background, dominated (10 kHz) by zodiacal light for the NUV and diffuse $(1 \mathrm{kHz})$ galactic light for the FUV.

Since the detectors are a critical element for GALEX, they were put through a life test to screen out ones that would potentially fail in the future. The flight tubes were placed in an oven to keep them at $30^{\circ} \mathrm{C}$ (the highest anticipated flight operational temperature) operated and illuminated with a UV light source. The tubes were run at full gain until the number of counts accumulated was about $10 \%$ of the counts expected during the GALEX mission. The NUV tube was operated at 70,000 counts/second for 8.7 days for a total of $5.3 \times 10^{10}$ counts. The FUV tube was exposed to 41,000 counts/second for 2 days for a total of $7.2 \times 10^{9}$ counts. The operational characteristics of the tubes (flat field, gain, and background) were monitored before, during and after the life test. All the tubes performed the same or better after the life test indicating that we had achieved satisfactory operational stability.

The event rate capability of the detector is established by two separate factors. One is the global rate capability as defined by the electronics processing chain and the other is the local event capability which is limited by the local charge throughput in the MCP pores. The global electronics processing time is of the order of a few microseconds, so that given the random arrival of photons will experience a $10 \%$ event loss at about $20 \mathrm{kHz}$. With the dead time calibration curve the event losses may be easily corrected to establish accurate photometry. The local counting rate limits are different for the FUV and NUV detectors, but is established by the input response to stellar "point" images. This is due to the effective resolution/spot size difference discussed below. The rate at which $10 \%$ of the incoming events are lost is $\sim 97$ 
events/sec/spot and 420 events/sec/spot for the FUV and NUV detectors respectively. Again it is possible to compensate for the event losses by employing a dead time correction calibration.

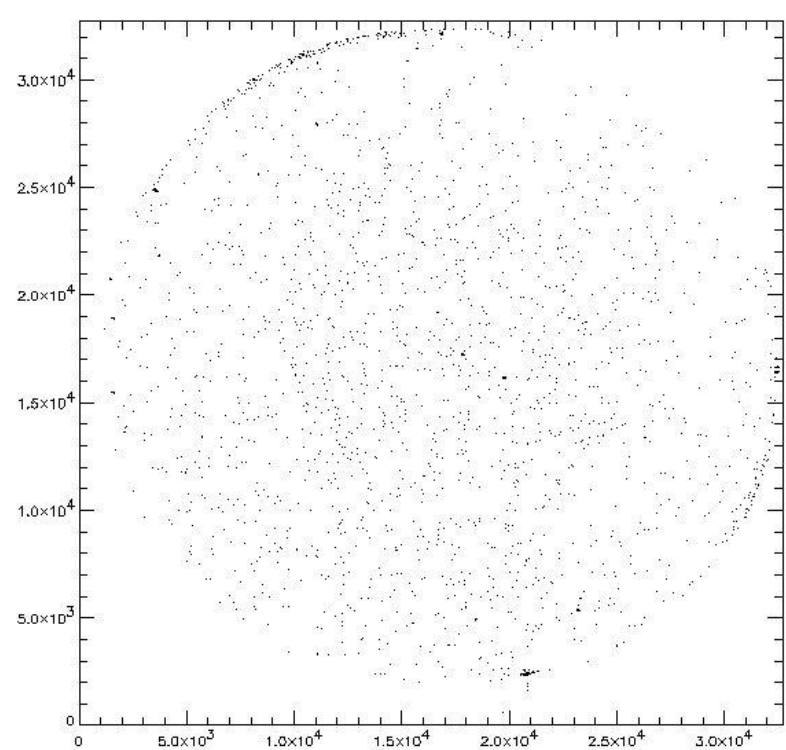

Figure 5. Pre delivery background image of the NUV tube at 5200 volts.

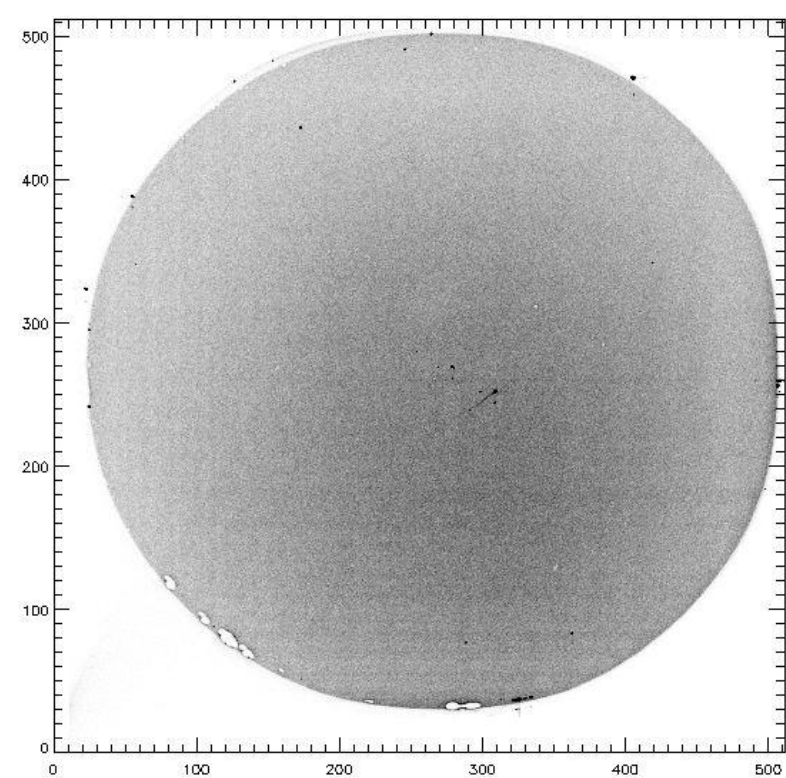

Figure 6. NUV flat field image taken at 5200 volts.

The detector flat field response maps measure the uniformity of the response of the tube to photons. They also can be used to measure the uniformity of the gain of the tube. Figure 6 shows the flat field image for the NUV tube taken at $2537 \AA$ with laboratory electronics before delivery. The NUV image is a 10 million event integration taken at 5200 volts, and shows few distinct features. The FUV flat field images show stripes in the image. These are caused by metal wires on the window, which function to establish a window-MCP field to enhance the QE. The wires detect long wavelength (visible) light that the photocathode does not. This effect is reduced significantly when just "in band light" is used.

The linearity of the detectors in the instrument was measured at JPL. A mask with $200 \mu$ m diameter pinholes was illuminated and placed at the focus of a duplicate telescope. The collimated light from the duplicate telescope was directed to the instrument. The instrument formed images of the pinhole mask on the detector and the data was analyzed to produce a correction map. The magnitude of the corrections is $<200 \mu \mathrm{m}$ over the central $50 \%$ of the detector area and is not caused by the tube itself but is due to the flight electronics non-linearities. Using the linearity corrections the position non-linearity can be corrected to about 15 microns ( $\sim 1$ arc-second) for about $3 / 4$ of the field of view.

The resolution and linearity of the tubes could only be measured at the Space Sciences Laboratory with laboratory electronics before the tubes were sealed. A mask with $10 \mu \mathrm{m}$ diameter pinholes spaced on a $1 \mathrm{~mm} \times 1 \mathrm{~mm}$ grid was placed in contact with the top MCP and UV light was used to illuminate the MCP through these pinholes. Figure 7 shows the resolution for the FUV tube as a function of gain, in both $\mathrm{X}$ and $\mathrm{Y}$ axes. At nominal operating gain of $\sim 1.5 \mathrm{x} 10^{7}$ the average full width at half maximum (FWHM) is about $38 \mu \mathrm{m}$. Given the size of the pinholes, the actual detector resolution with rack electronics is about $36 \mu \mathrm{m}$ FWHM. In the case of the FUV finished tube there will be an additional contribution due to the collection of photoelectrons from the cathode material on the MCP webbing, using the window-MCP field. The resolution of the NUV tube is also modified by the proximity focus of the photocathode on the window to MCP. Lateral spread of photoelectrons in this gap causes some blurring of the resolution. The resolution of these tubes in the instrument was measured in a similar manor but using a mask with $20 \mu \mathrm{m}$ pinholes. The resolution includes the resolution of both of the telescopes and the detector contribution. So it is an upper limit to the detector resolution. The measured resolution for the FUV channel was about $105 \mu \mathrm{m} \mathrm{80 \%} \mathrm{encircled} \mathrm{energy} \mathrm{(EE)} \mathrm{diameter.} \mathrm{The} \mathrm{collimator} \mathrm{and} \mathrm{telescope} \mathrm{add} \mathrm{about} 41 \mu \mathrm{m}$ due to astigmatism and the pinholes add about $20 \mu \mathrm{m}$. Therefore the derived resolution of the FUV tube with flight electronics is about $53 \mu \mathrm{m}$ FWHM. The measured resolution of the NUV channel was $135 \mu \mathrm{m} 80 \%$ EE diameter. Therefore the derived resolution of the NUV tube with flight electronics is about $78 \mu \mathrm{m}$ FWHM. 


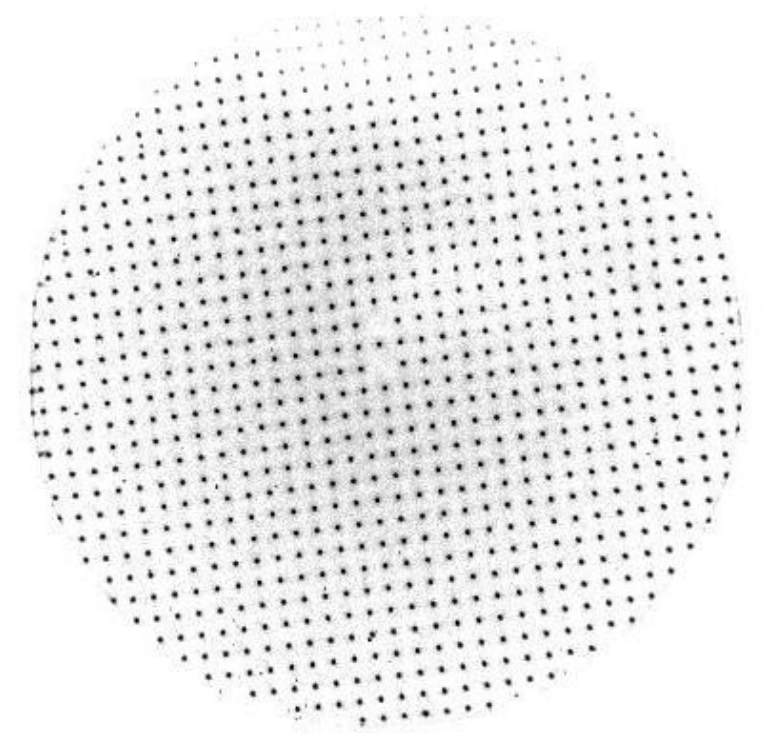

Figure 7. Image used for FUV linearity assessment.

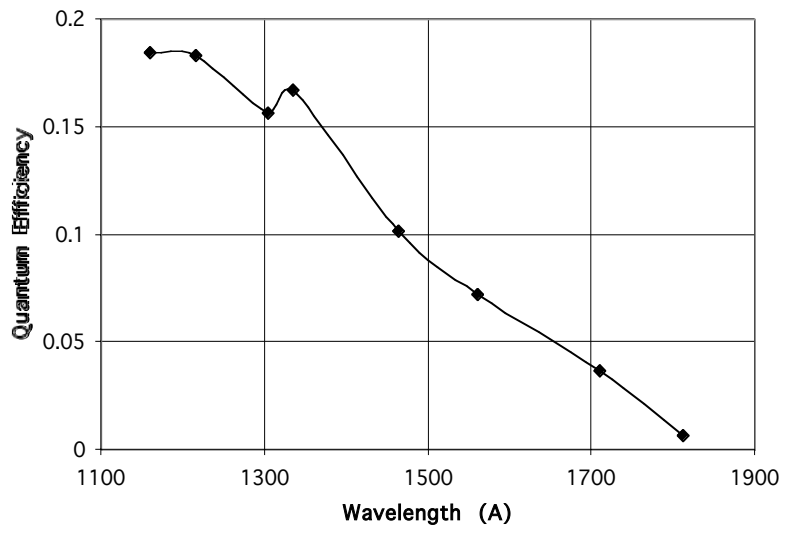

Figure 9. Quantum efficiency of the FUV tube + window, CsI photocathode on MCP.

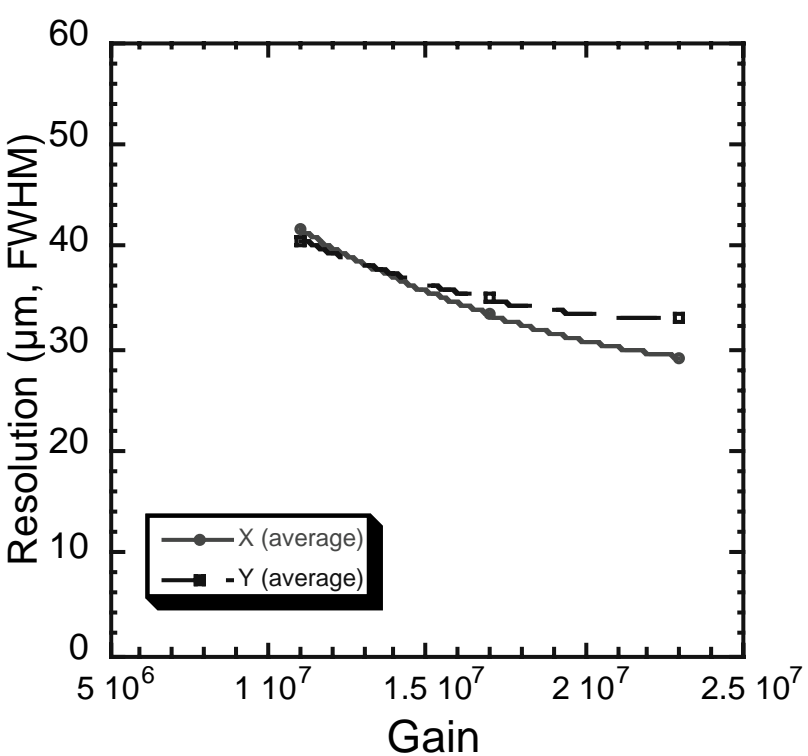

Figure 8. FUV resolution prior to window seal measured with a mask in contact with the top MCP.

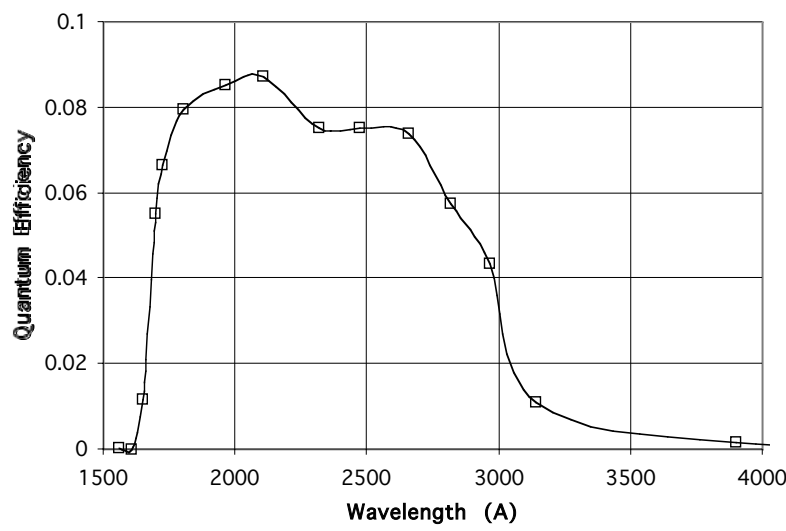

Figure 10. Quantum efficiency of the NUV tube, + window, $\mathrm{Cs}_{2} \mathrm{Te}$ photocathode on window.

Figure 9 shows the QE of the FUV tube and Figure 10 shows the QE of the NUV tube, both of which comprise the final QE that includes the transmission of the windows, QE of the cathode and detection efficiency of the MCP's. In addition, the relative QE was measured at several positions on the detector and the QE variations were $\sim 13 \%$ RMS for the FUV detector and $\sim 8 \%$ RMS for the NUV detector. The QE of the NUV tubes (see Figure 10) is very similar to other MCP-based detectors on orbit, such as the STIS MAMA on board the Hubble Space Telescope, and is typical for Cs $\mathrm{S}_{2}$ Te semitransparent photocathodes. It is interesting to note that significant improvements $(>50 \%$ QE) may be achieved in future instruments by the use of newly developed ${ }^{8} \mathrm{GaN}$ photocathodes. The QE of the FUV CsI cathode is lower than we have achieved on a number of other missions. In large part this is due non-optimization of the baking and UV scrubbing process needed to seal the tube after the cathode deposition has occurred. In principal, modification of the processing sequence would have yielded higher QE values. 


\section{SCIENCE OBSERVATIONS WITH GALEX}

\subsection{Scientific Motivation for GALEX}

The rest UV provides a powerful tool for measuring and understanding star formation in galaxies at all epochs, a fact underscored by the recent discovery and study of a population of star-forming Lyman Break galaxies ${ }^{8}$ at a red-shift of $\mathrm{z} \sim$ 3. Unfortunately technological obstacles have slowed progress in mapping the UV sky, a process that started over 30 years ago with the launch of the Orbital Astronomical Observatory ${ }^{9}$, OAO-2. However, with the recent advances in developing large format high sensitivity UV detectors and efficient wide-field UV optics it has been possible to design a space mission that addresses the following three primary science goals within the cost cap of a Small Explorer mission budget. The primary goal of the GALEX mission will be to provide a calibration of UV and galaxy star formation rate that accounts for extinction, starburst history, initial mass function and metallicity. This calibration is to be obtained over a wide range of star formation environments such that the relationships can also be applied to galaxies at cosmic epochs where star formation may assume very different characteristics.

The second goal of the GALEX mission is to use the UV imaging survey data to determine the cosmic star formation history over the red-shift range $0<\mathrm{z}<1.5$ (i.e. over the past 9 Gyrs!), and to determine its dependence on environment, mass, morphology, merging and star formation modality. These data will then be compared with similar galaxy survey data being obtained at longer wavelengths (e.g. in the infra-red by the Spitzer Observatory) which is relevant for the rest wavelengths of UV-bright galaxies at redshifts $1.5<\mathrm{z}<20$ (i.e. the first 4 Gyrs). Finally the third science goal of the GALEX mission is to obtain large statistical samples and detailed studies of nearby galaxies (such as Andromeda) to obtain predictive models of star formation rates in the Local Group. Serendipitously GALEX will also observe a large variety of foreground galactic stellar (UV bright) sources ranging from hot white dwarf stars in the galactic halo to late-type flare stars located within 20pc of the Sun.

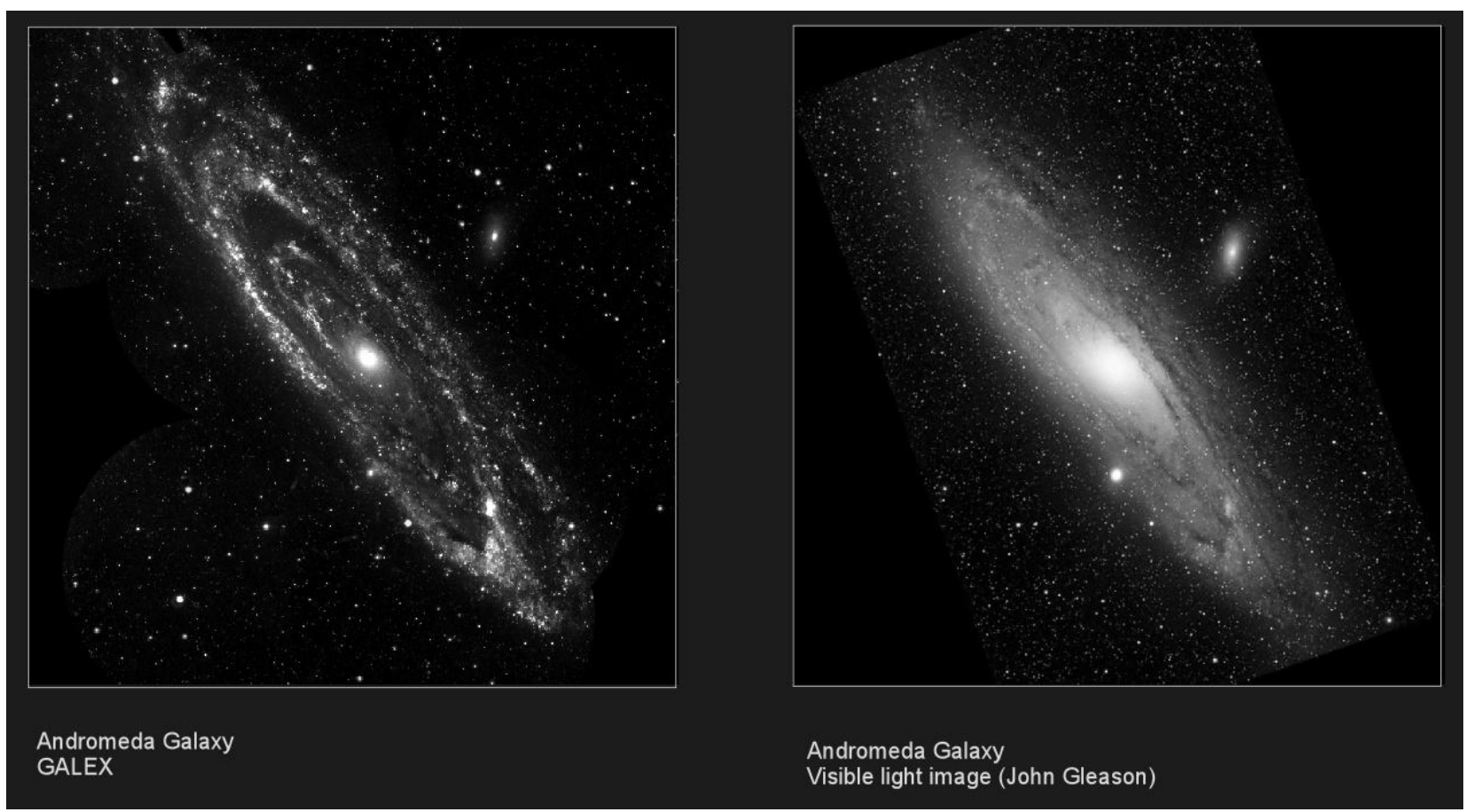

Figure 11. Combined bandpass GALEX image of M31

Figure 12. Visible light image of M31.

The aforementioned prime science goals of the GALEX mission are achieved through both far and near UV widefield deep imaging, supplemented by wide-field low spectral resolution UV spectroscopy. Early results from the mission include the detection of extended star formation in numerous disk galaxies, the detection of luminous UV analogs to the Lyman Break Galaxies in the local universe and the wide-spread detection of star formation in red galaxies. In addition to extra-galactic studies GALEX is also supporting a wide range of other astrophysical investigations such as the discovery of extended gas nebulosity around the dwarf nova Z Cam, the real-time detection of a UV Ceti-type flare event and the 
observation of an RR Lyrae star over its entire light-curve. All of these topics will be covered in detailed refereed papers contained in a special GALEX edition of the Astrophysical Journal Letters to be published in late 2004.

\subsection{Early GALEX Observations}

One of the first extra-ordinary images to be released by GALEX was the far UV image of the entirety of the Andromeda galaxy (M31) shown in Figure 11 which covers an area of $\sim 1.5^{\circ} \mathrm{x} 1.5^{\circ}$ on the sky. This image highlights the UV emission from the hot young high mass OB-type stars that trace out the spiral arms of this galaxy where star formation is occurring. Adjacent to this UV image is that of the same galaxy recorded in visible light (Figure 12), in which the star forming regions are 'hidden' by the interstellar gas and dust of M31. These UV images have been used by Rey et al (2003) to investigate UV bright stars in over one hundred globular clusters surrounding M31. Early results suggest that there are no great differences between the stellar content of Andromeda and our own Milky Way galaxy.

During the first year of operation GALEX has completed its Nearby Galaxy Survey (NGS) of $\sim 200$ targets that are also currently being observed by the Spitzer Infrared nearby Galaxy Survey (SINGS). By combining both of these sets of survey data with that of ground-based Sloan Digital Sky Survey observations many fundamental plots of galaxy colors, luminosity and surface brightness can be made, which have revealed many new correlations between their UV and optical/IR properties. The nearby galaxy, M101 (seen in Figure 13) has been selected for in-depth study by Bianchi et al (2004), in which photometry of all the compact star clusters (down to a $\sim 150 \mathrm{pc}$ scale) revealed by the GALEX UV imaging have been compared with synthetic model UV colors derived for a single star-forming burst of stellar population. The fitting of the observed UV magnitudes with model magnitudes has provided ages, intrinsic luminosities and the current and initial star cluster masses. These data suggest that there is a younger stellar population of stars located towards the outer parts of the M101 disk.

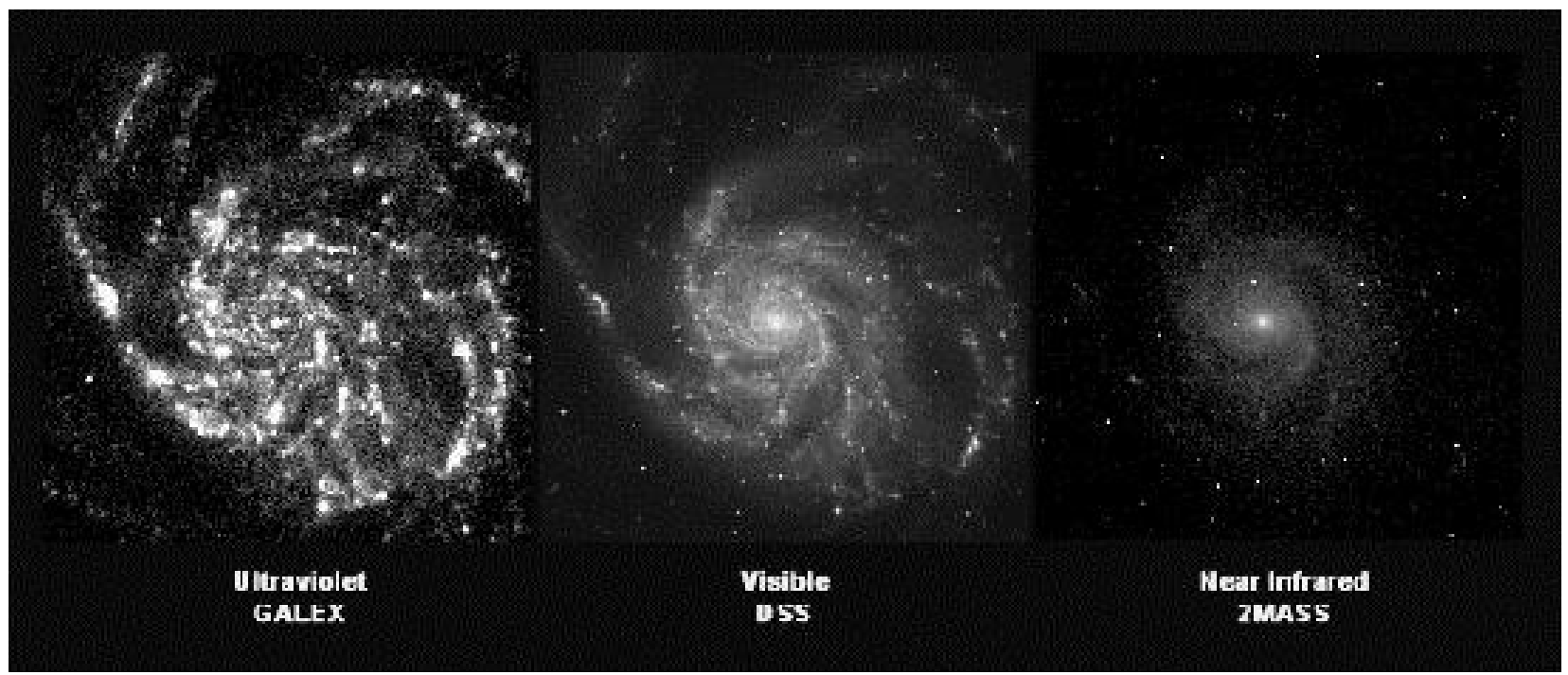

Figure 13. Comparison of GALEX image of M101 with visible light and near IR images

An unexpected discovery by GALEX was the detection of a rare population of low redshift starburst galaxies with physical properties remarkably similar to the high-redshift Lyman Break Galaxies (LBGs). This new local galaxy population resemble LBGs in terms of their size $(2-5 \mathrm{kpc})$, their UV luminosity ( $\mathrm{L}>2 \times 10^{10} \mathrm{~L}_{\text {sun }}$ ), metalicity, dust content and color ${ }^{1}$.

GALEX is also accumulating a catalog of $\sim 100,000$ QSOs that can be recognized easily from UV/optical color-color diagrams. Within this large QSO sample it is hoped to find several hundreds of QSO pairs with less than 10Mpc separation. At $\mathrm{z}=1$ such pairs will provide the opportunity to measure large scale 3-D correlations in the Lyman- $\alpha$ forest, which will be a powerful constraint on the dark energy equation of state and models of cosmic structure. The very brightest of the newly discovered QSOs will be observed with higher spectral resolution by the HST-STIS instrument in order to study the re-ionization of HeII in the inter-galactic medium, which is a direct measure of the physical conditions in the inter-galactic medium.

GALEX observations of sources within our own galaxy have also provided several surprises. UV images of the dwarf nova star Z Camelopardalis (Z Cam) have revealed several distinct shell-like filamentary structures of gaseous ejecta up to 20 arc minutes in radius (Figure 14). This is the first evidence that dwarf novae may undergo eruptions similar to those of 
classical stellar novae, and subsequent analysis of the ejected material surrounding Z Cam should reveal signs of thermonuclear processing.

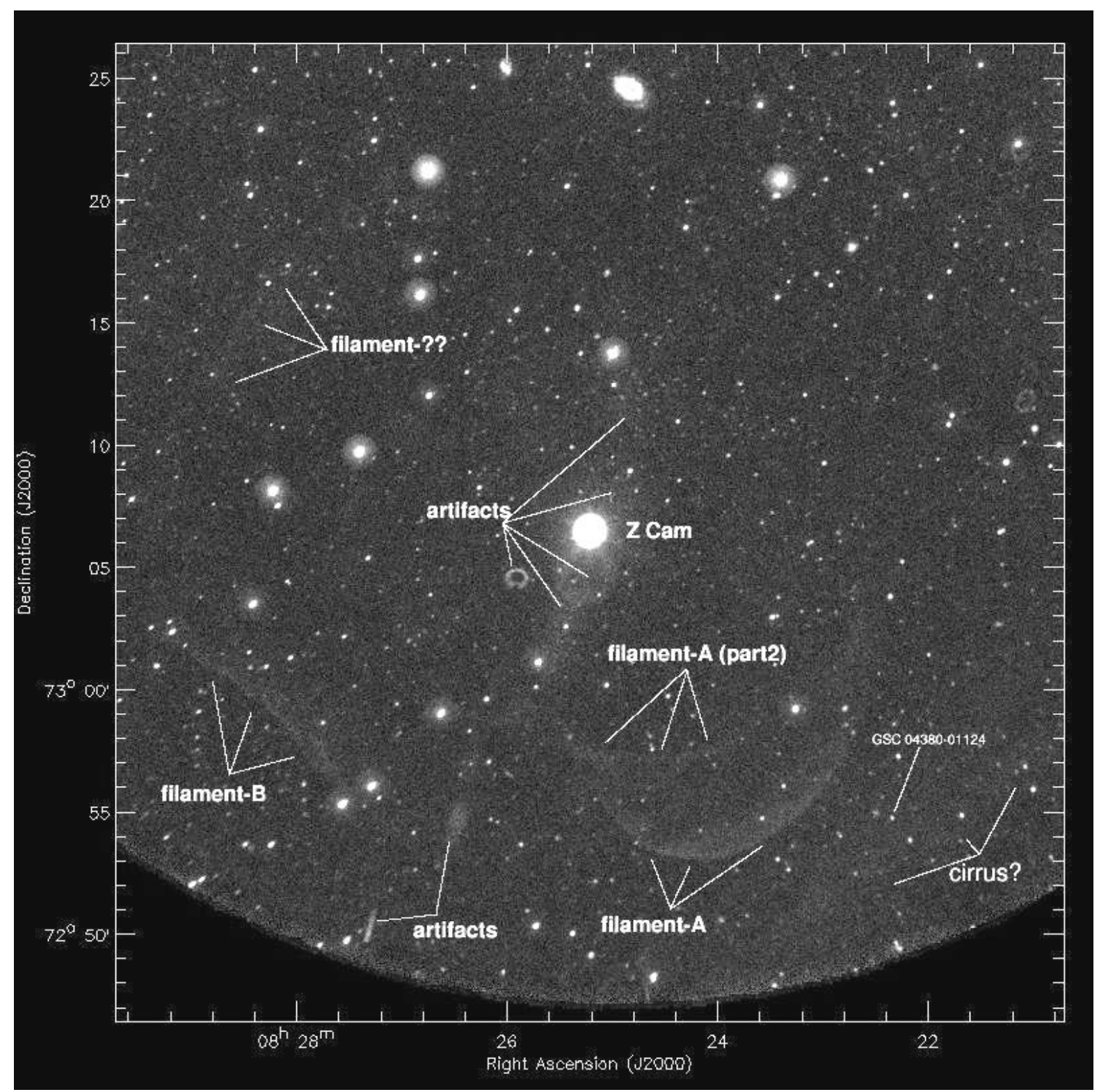

Figure 14. Arcs and loops of filamentary gas ejecta surrounding the dwarf nova-type star Z Cam

In Figure 15. we show the first complete near and far UV light-curves for the RR Lyrae star, ROTSE-I J$143753.84+345924.8$ as measured by GALEX ${ }^{10}$. For comparison purposes we also show the far smaller range of intrinsic variability in the visible region (as measured by the ROTSE instrument), which is to be compared with a UV magnitude variation of over 5 magnitudes in the far UV region. These UV data have been used with stellar model atmospheres to derive both the temperature and metal content of the atmosphere of this star. 


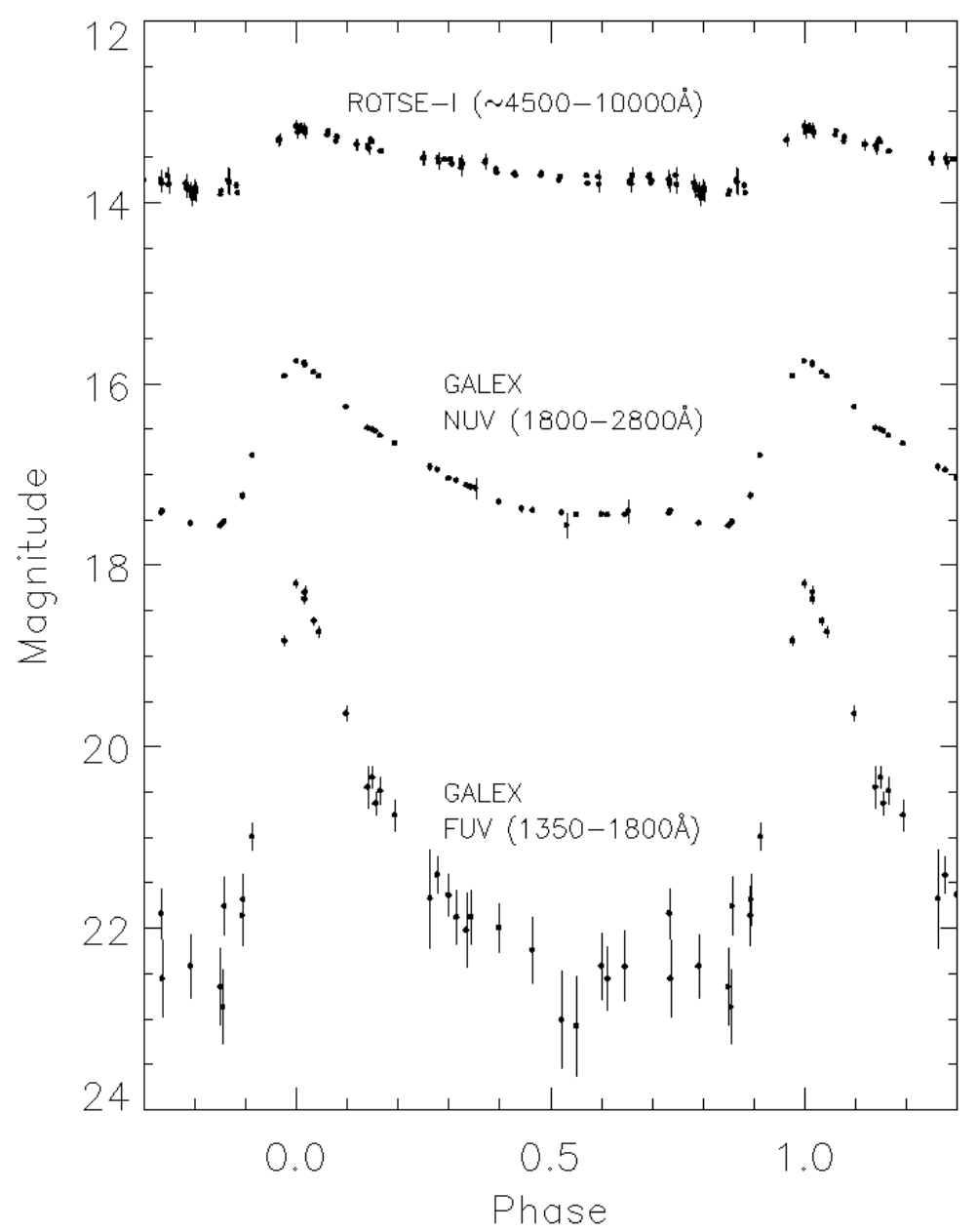

Figure 15. GALEX FUV and NUV light curves for the ROTSE RR Lyrae star, together with corresponding visible light curves. Note the far larger magnitude variation in the UV wavelength domain.

GALEX is also collecting wide-field spectroscopic data on many of the survey fields initially recorded in the UV imaging mode. Due to the complexity of the data reduction routines that are required to be applied to the raw data (taken at several grism angles per spectral image) we are only just obtaining fully calibrated UV spectra that have been run through the GALEX scientific data processing pipe-line. In Figure 16 we show a typical detector spectral image obtained during the Wide Spectroscopic Survey of the ELAISS-1 galaxy field recorded at one position angle of the grism. In this image we clearly see the 'streaks' that are associated with the dispersed FUV/NUV low resolution spectra of the brightest UV sources in this field. In Figure 17 we show an ultraviolet spectrum of one of the sources in this field, a $\mathrm{z}=0.5$ quasar that shows its red-shifted Ly- $\alpha$ emission line at $1820 \AA$ and the CIV line red-shifted to $2325 \AA$.

\section{ACKNOWLEDGEMENTS}

GALEX (Galaxy Evolution Explorer) is a NASA Small Explorer, launched in April 2003. We gratefully acknowledge NASA's support for construction, operation, and science analysis for the GALEX mission, developed in cooperation with the Centre National d'Etudes Spatiales of France and the Korean Ministry of Science and Technology. The grating, window, and aspheric corrector were supplied by France. We acknowledge the dedicated team of engineers, technicians, and administrative staff from JPL/Caltech, Orbital Sciences Corporation, University of California, Berkeley, Laboratory Astrophysique Marseille, and the other institutions who made this mission possible. 


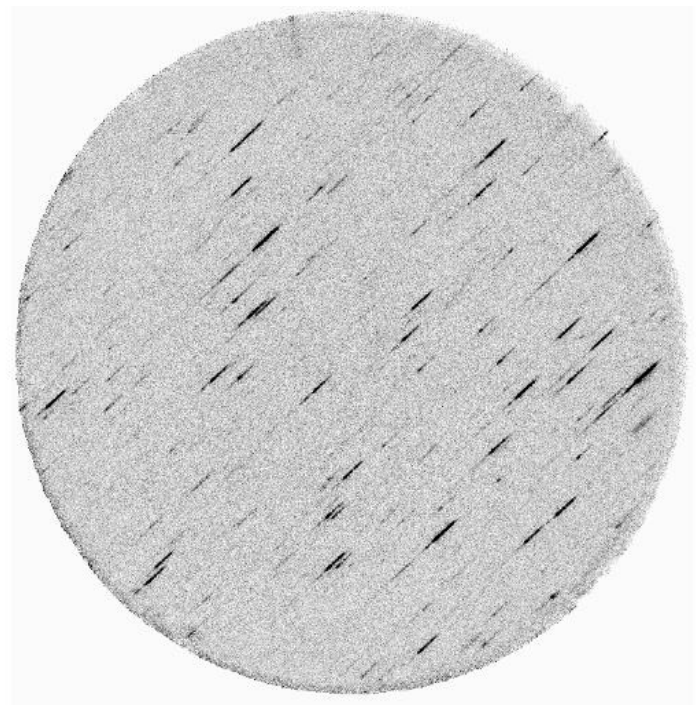

Figure 16: GALEX wide-field slit-less spectroscopic Observations of the ELAISS-1 field.

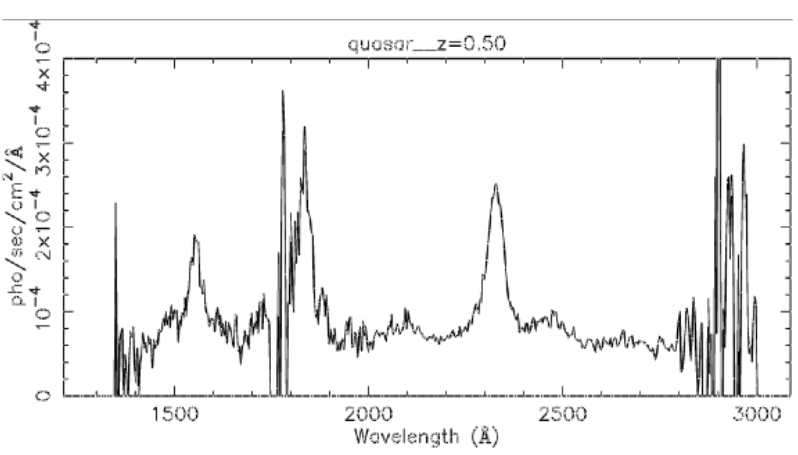

Figure 17: GALEX UV spectrum of a $\mathrm{z}=0.5$ quasar

\section{REFERENCES}

1. C. Martin, et al.,"The Galaxy Evolution Explorer a space ultraviolet survey mission", Accepted by Ap. J. Lett. 2004.

2. C. Martin, et al., "The Galaxy Evolution Explorer", Proc. SPIE, 4854, pp. 2002.

3. P. Morrissey, et al., "The on orbit performance of the Galaxy Evolution Explorer", Accepted by Ap. J. Lett. 2004.

4. O. Siegmund, P. Jelinsky, S. Jelinsky, J. Stock, J. Hull, D. Doliber, J. Zaninovich, A. Tremsin, K. Kromer, "Highresolution cross delay line detectors for the GALEX mission", Proc. SPIE, 3765, pp. 429-440, 1999.

5. P. Jelinsky, P. Morrissey, James Malloy, S. Jelinsky, and O. Siegmund, C. Martin, D. Schiminovich, K. Forster, T. Wyder \& P. Friedman, "Performance Results of the GALEX XDL detectors", Proc. SPIE, 4854, pp.233-240, 2002.

6. O. H. Siegmund, J. M. Stock, D. R. Marsh, M. A. Gummin, R. Raffanti, J. Hull, Jeffrey, G. A. Gaines, B. Y. Welsh, B. Donakowski, P. N. Jelinsky, T. Sasseen, J. L. Tom, B. Higgins, T. Magoncelli, J. W. HamiltonS. J. Battel, A. I. Poland, M. D. Jhabvala, K. Sizemore, J. Shannon, "Delay Line Detectors for the UVCS and SUMER Instruments on the SOHO Satellite", Proc. SPIE, 2280, pp. 89-100, 1994.

7. J. M. Stock, O. H. Siegmund, J. S. Hull, K. E. Kromer, S. R. Jelinsky, H. D. Heetderks, M. L. Lampton, S. B. Mende, "Cross-delay-line microchannel plate detectors for the Spectrographic Imager on the IMAGE satellite" Proc. SPIE, 3445, 407, 1998.

8. C. Steidel, M. Giavalisco, M. Pettini, M. Dickinson and K. Aldelberger, "Spectroscopic confirmation of a population of normal star-forming galaxies at redshifts z $>$ 3", Ap. J. Lett. 462, L17, 1996.

9. A. Code, T. Houck, J. McNall, R. Bless. and C. Lillie, "Ultraviolet photometry from the Orbiting Astronomical Observatory I: Instrumentation and operation.”, Ap. J. 161, 377, 1970.

10. J. Wheatley. et al., "Large amplitude UV variations in the RR Lyrae star ROTSE-I J143753.84+345924.8", Accepted by Ap. J. Lett, 2004. 